\title{
Impact of Glycosylated Hemoglobin on the Prognosis of Patients with Acute Ischemic Stroke Treated with Arterial Thrombolysis
}

\author{
Wentong Ling, ${ }^{1}$ Qiong Chen, ${ }^{2}$ Pu Huang, ${ }^{1}$ Dengke Han, ${ }^{2}$ Wenjun $\mathrm{Wu}^{1}$ \\ ${ }^{1}$ Department of Neurology and ${ }^{2}$ Department of Clinical Medicine, Zhongshan City People's Hospital, Zhongshan 528400, China
}

\section{ABSTRACT}

Background: To investigate the impact of glycosylated hemoglobin (HbA1c) on the prognosis of patients with acute ischemic stroke (AIS) treated with intra-arterial thrombolysis (IAT).

Methods: The clinical data of 136 patients with AIS treated with IAT at the Zhongshan City People's Hospital were retrospectively analyzed. The patients were divided into a high HbA1c group (HHbA1c) $(\geq 6.5 \%)$ and a normal HbA1c group (NHbA1c) ( $<6.5 \%)$. According to National Institutes of Health Stroke Scale (NIHSS) score after thrombolysis, patients were divided into a good prognosis group (GP) ( $\geq 4$ or $<4$ points reduction) and a poor prognosis group (PP) ( $\leq 4$ or $>4$ points reduction).

Results: There were significant differences in the HbA1c and glucose levels, NIHSS scores at admission and at discharge, complication rates, and mortality rates between groups HHbA1c and NHbA1c $(P<.05)$ and between groups GP and PP $(P<.05)$. The multivariate logistic regression analysis showed that HbA1c level (odds ratio [OR] 0.717; 95\% confidence interval [CI] 0.545 to 0.889 ) and NIHSS score at admission (OR $0.894 ; 95 \%$ CI 0.814 to 0.982 ) were risk factors for neurological improvement in IAT-treated patients with AIS.

Conclusions: HbA1c level is associated with neurological function improvement in IAT-treated patients with AIS and can be used as a serological indicator of poor prognosis.

\section{INTRODUCTION}

Stroke has become the leading cause of disability and mortality in China. Diabetes is recognized as one of the risk factors for stroke and is closely related to the occurrence and recurrence of acute ischemic stroke (AIS) [O'Donnell 2016]. Blood glucose and glycosylated hemoglobin (HbA1c) levels are associated with poststroke prognosis, and the latest guidelines recommend strict glycemic control in patients with AIS [Jauch 2013]. The HbA1c level can reflect the blood glucose status before stroke [Roquer

Received Feburay 13, 2020; accepted April 8, 2020.

Correspondence: Wenjun Wu, Department of Neurology, Zhongshan City People's Hospital, No. 2 Sunwen Road, Zhongshan 528400, China; +86-0760-89880204; fax:+86-0760-88823566 (e-mail: wenjunwudoc@163.com).
2014]. The poststroke blood glucose status [Desilles 2013], that is, blood glucose level at admission, has been widely investigated for its impact on the occurrence and prognosis of AIS. Studies have shown that blood glucose level is correlated with the severity of stroke, as well as with the types of stroke caused by macrovascular diseases and cardiogenic embolism, but it has no correlation with stroke caused by small blood vessel diseases [Lee 2018]. High HbA1c levels are negatively correlated with neurological function recovery 3 months after small arterial occlusion [Gao 2016]. In patients with a history of diabetes or poststroke hyperglycemia, intravenous thrombolysis for AIS results in a poor prognosis, hemorrhagic transformation, or even death [Pandolfi 2001]. Intravenous thrombolysis has a low revascularization rate in patients with acute hyperglycemia [Kamouchi 2011], which normally predicts poor prognosis, increased intracranial hemorrhage [Ahmed 2010], and high mortality [Jia 2011].

In the PROACT II trials, diabetes and hyperglycemia showed no association with the prognosis of intra-arterial thrombolysis (IAT) [Wechsler 2003]. Hyperglycemia is an independent predictor of symptomatic intracranial hemorrhage [Hallevi 2009]. IAT is the direct application of fibrinolytic drugs to the responsible vessels; thus, the vascular recanalization rate is high, but the occurrence of hemorrhagic transformation and hyperperfusion injury after thrombolytic therapy is inevitable in some patients. Therefore, it is important to evaluate arterial thrombolytic therapy and postoperatively control blood glucose levels, thus improving prognosis. The relationship between HbAlc levels and the prognosis of AIS after IAT is unknown. The aim of this study was to investigate the impact of HbA1c on the prognosis of patients with AIS treated with IAT.

\section{METHODS}

This study was conducted in accordance with the Declaration of Helsinki and with approval from the Ethics Committee of Zhongshan City People's Hospital. Written informed consent was obtained from all participants.

\section{Inclusion and Exclusion Criteria}

The inclusion criteria for IAT were as follows: $(1)<80$ years of age; (2) digital subtraction angiography-confirmed occlusion in the neurological dysfunction-corresponding vessels; (3) no intracranial infarction, cerebral hemorrhage, or 
Table 1. Comparison of Clinical Data between Groups HHbA1c and NHbA1c

\begin{tabular}{|c|c|c|c|c|}
\hline $\operatorname{Sex}(M / F)^{*}$ & $41 / 28$ & $35 / 32$ & 0.711 & .049 \\
\hline Drinking $(\mathrm{Y} / \mathrm{N})$ & $11 / 58$ & $8 / 59$ & 0.435 & .501 \\
\hline Smoking $(\mathrm{Y} / \mathrm{N})$ & $16 / 53$ & $13 / 54$ & 0.290 & .590 \\
\hline Hypertension $(\mathrm{Y} / \mathrm{N})$ & $48 / 21$ & $52 / 15$ & 1.131 & .288 \\
\hline Heart disease $(\mathrm{Y} / \mathrm{N})$ & $20 / 49$ & $23 / 44$ & 0.449 & .503 \\
\hline Systolic blood pressure $(\mathrm{mmHg})$ & $155.11 \pm 23.04$ & $157.71 \pm 24.20$ & 0.734 & .806 \\
\hline Diastolic blood pressure $(\mathrm{mmHg})$ & $86.21 \pm 17.21$ & $91.04 \pm 13.91$ & 1.874 & .251 \\
\hline Onset time $(h)$ & $6.67 \pm 1.11$ & $6.30 \pm 1.49$ & 1.918 & .264 \\
\hline $\mathrm{HCY}(\mu \mathrm{mol} / \mathrm{L})$ & $11.45 \pm 3.61$ & $13.00 \pm 3.45$ & 0.859 & .355 \\
\hline Glucose $(\mathrm{mmol} / \mathrm{L})^{*}$ & $5.01 \pm 0.45$ & $8.53 \pm 2.42$ & 11.761 & .000 \\
\hline $\mathrm{HbA} 1 \mathrm{C}(\%)^{*}$ & $5.41 \pm 0.31$ & $7.11 \pm 1.18$ & 7.265 & .000 \\
\hline NIHSS score on admission* & $6.04 \pm 3.42$ & $7.11 \pm 1.19$ & 7.265 & .011 \\
\hline NIHSS score on discharge* & $2.11 \pm 0.27$ & $6.19 \pm 1.18$ & 3.376 & .008 \\
\hline Complication $(\mathrm{Y} / \mathrm{N})^{*}$ & $1 / 68$ & $10 / 57$ & 7.187 & .007 \\
\hline NIHSS score improvement $(\mathrm{Y} / \mathrm{N})^{*}$ & $62 / 7$ & $49 / 18$ & 6.334 & .012 \\
\hline Death $(\mathrm{Y} / \mathrm{N})^{*}$ & $0 / 69$ & $4 / 63$ & 5.266 & .022 \\
\hline
\end{tabular}

*Intergroup comparison, $P<.05$.

other intracranial diseases on the initial cranial computed tomography (CT); (4) within 6 hours of stroke onset and within 12 hours of the onset of basilar artery occlusion; and (5) consent for treatment (the patient's family agreed and signed the informed consent). The exclusion criteria were as follows: (1) NIHSS score $\geq 22$ points; (2) bleeding tendency; (3) history of a major surgery or trauma within 2 months; (4) history of a major organ dysfunction or failure; and (5) pretreatment systolic blood pressure $\geq 180 \mathrm{mmHg}$ or diastolic blood pressure $\geq 110 \mathrm{mmHg}$. Each patient's vital signs were monitored after surgery, and repeat head CT was performed within 24 hours after surgery to monitor for complications, such as cerebral hemorrhage. National Institutes of Health Stroke Scale (NIHSS) scoring was used to assess the degree of neurological deficit in patients with stroke, and the baseline value was used to regularly assess the severity of the stroke as well as the treatment effect. Patients with a baseline assessment $>16$ points were more likely to die, but those with a baseline assessment $<6$ points were likely to recover well; every increase in the score by 1 point reduced the possibility of a good prognosis by $17 \%$. The score ranged from 0 to 42 points; the higher the score, the more severe the nerve damage.

\section{Study Data}

We retrospectively analyzed the clinical data of 136 patients with AIS treated with IAT in our hospital from January 2015 to January 2018, including 76 males and 60 females, with a mean $( \pm \sigma)$ age of $62.25 \pm 13.17$ years. The patients were divided into a high HbA1c group (HHbA1c) (n $=67, \mathrm{HbA} 1 \mathrm{c} \geq 6.5 \%$ ) and a normal HbA1c group (NHbA1c) $(\mathrm{n}=69, \mathrm{HbA} 1 \mathrm{c}<6.5 \%)$. According to NIHSS score after thrombolysis, patients were divided into a good prognosis group (GP) $(\mathrm{n}=111, \geq 4$ or $<4$ points reduction) and a poor prognosis group $(\mathrm{PP})(\mathrm{n}=25, \leq 4$ or $>4$ points reduction).

\section{Observation Indexes}

The patients' basic data, including sex, age, HbA1c (HPLC, PRIMUS Ultra2 glycosylated hemoglobin analyzer); glucose, homocysteine (HCY), total cholesterol (TC), and triglyceride (TG) levels; and laboratory indexes were measured using a German Roche Cobas8000 automatic biochemical analyzer. The treatment onset time; length of hospital stay; history of smoking, drinking, heart disease, stroke, or hypertension; NIHSS score at admission and discharge; and complications (bleeding, hyperperfusion, or reinfarction) were also recorded. The surgical 
Table 2. Comparison of Clinical Data between Group GP and Group PP

\begin{tabular}{|c|c|c|c|c|}
\hline $\operatorname{Sex}(M / F)^{*}$ & $62 / 49$ & $14 / 11$ & 0.154 & .832 \\
\hline Drinking $(\mathrm{Y} / \mathrm{N})$ & $15 / 96$ & $4 / 21$ & 0.105 & .446 \\
\hline Smoking $(\mathrm{Y} / \mathrm{N})$ & $26 / 85$ & $10 / 15$ & 0.206 & .564 \\
\hline Hypertension $(\mathrm{Y} / \mathrm{N})$ & $84 / 27$ & $20 / 5$ & 0.212 & .645 \\
\hline Heart disease $(\mathrm{Y} / \mathrm{N})$ & $36 / 75$ & $7 / 18$ & 0.185 & .667 \\
\hline Systolic blood pressure $(\mathrm{mmHg})$ & $156.55 \pm 22.07$ & $155.68 \pm 29.86$ & -0.139 & .891 \\
\hline Diastolic blood pressure $(\mathrm{mmHg})$ & $88.77 \pm 14.69$ & $87.80 \pm 20.38$ & -0.226 & .823 \\
\hline Onset time (h) & $6.92 \pm 3.03$ & $6.26 \pm 4.04$ & 0.363 & .719 \\
\hline $\mathrm{HCY}(\mu \mathrm{mol} / \mathrm{L})$ & $11.43 \pm 3.90$ & $15.67 \pm 4.22$ & 0.999 & .327 \\
\hline Glucose $(\mathrm{mmol} / \mathrm{L})^{*}$ & $6.46 \pm 2.32$ & $8.14 \pm 2.69$ & 2.885 & .007 \\
\hline $\operatorname{HbA1c}(\%)^{*}$ & $6.04 \pm 1.39$ & $7.21 \pm 2.06$ & 2.740 & .010 \\
\hline NIHSS score on admission* & $6.38 \pm 4.29$ & $9.04 \pm 4.56$ & 2.656 & .012 \\
\hline NIHSS score on discharge* & $1.90 \pm 1.75$ & $14.12 \pm 6.68$ & 4.805 & .000 \\
\hline Complication $(\mathrm{Y} / \mathrm{N})^{*}$ & $5 / 106$ & $6 / 19$ & 12.049 & .001 \\
\hline Death $(\mathrm{Y} / \mathrm{N})^{*}$ & $0 / 111$ & $4 / 21$ & 18.298 & .000 \\
\hline
\end{tabular}

*Intergroup comparison, $P<.05$.

treatment in all patients was performed by the neurosurgeons of the study, and neurological function scoring was performed by a neurology resident or a physician with a higher professional title.

\section{Statistical Analysis}

SPSS 13.0 statistical software was used. The measurement data are expressed as $\bar{x} \pm \mathrm{s}$. The $\mathrm{t}$ test was used for intergroup comparisons, and the $\chi^{2}$ test for the count data comparison. Multivariate linear logistic regression analysis was used to analyze the relationship between multiple risk factors and neurological function changes, with $P<.05$ being considered to indicate statistical significance.

\section{RESULTS}

Comparison of the Clinical Data between Groups HHbA1c and $\mathrm{NHb}$ A1c

The comparison of the clinical data between groups HHbA1c and NHbA1c is shown in Table 1. The values of glucose, HbA1c, complication rate (16\%), mortality rate $(6 \%)$, NIHSS score at admission, NIHSS score at discharge, and NIHSS score improvement were higher in group HHbA1c than those in group NHbA1c $(P<.05)$; the complications included 5 cases of hemorrhage, 4 cases of hyperperfusion edema, and 2 cases of reinfarction.

Comparison of the Clinical Data between Groups GP and PP

The comparison of the clinical data between groups GP and PP is shown in Table 2. The values of glucose, HbA1c, complication rate, mortality rate, NIHSS score at admission, and NIHSS score at discharge were higher in group PP than those in group GP $(P<.05)$.

\section{Multivariate Logistic Linear Regression Analysis}

The results of the multivariate logistic linear regression analysis targeting the relationship between the NIHSS score at discharge and multiple risk factors are shown in Table 3. HbA1c levels (odds ratio [OR] 0.096, 95\% confidence interval 0.545 to 0.889 ) and NIHSS score at admission (OR 0.894 , $95 \%$ CI 0.814 to 0.982 ) were determined to be risk factors for neurological function changes after IAT $(P<.05)$.

\section{DISCUSSION}

In patients with AIS with an onset time of $<4.5$ hours, intravenous thrombolysis should be performed immediately if there are no contraindications for systemic thrombolysis. 
Table 3. Multivariate Logistic Linear Regression Analysis of Relationship between Neurological Function Changes and Multiple Risk Factors

\begin{tabular}{lcccccc}
\hline Variable & $\beta$ & SE & Wald & P & OR \\
\hline Glucose & -0.046 & 0.030 & 2.313 & .128 & 0.955 & 0.900 or 1.013 \\
HbA1c & -3.636 & 0.125 & 8.433 & .004 & 0.717 & 0.545 or 0.899 \\
NIHSS on admission & -0.112 & 0.048 & 5.469 & .019 & 0.894 & 0.814 or 0.928 \\
Constant & 5.301 & 1.005 & 25.495 & .000 & 200.505
\end{tabular}

If there is no significant improvement in neurological function, such patients should be promptly converted to interventional therapy. In patients with more severe strokes (NIHSS score $>10$ ) and with a time window between 4.5 and 6 hours, systemic thrombolysis should be rapidly converted to neurointervention.

The current study was a single-center retrospective study, and in the selected patients, recanalization was achieved after IAT. Patients requiring stent thrombectomy or stenting for recanalization were not included in the study. Therefore, the recanalization rate of blood vessels was not compared. In this study, we included patients with AIS treated with IAT, and some of the patients had an unclear history of diabetes or could not be diagnosed as having diabetes. The glucose value measured at admission reflects the stroke-stimulated blood glucose level, whereas the HbAlc value is a stable and accurate indicator reflecting long-term blood glucose control before stroke. All enrolled patients had an admission time within 6 hours of stroke onset, and the average hospital stay length was approximately 14 days.

The results show that there was a statistically significant difference in the NIHSS scores at admission and at discharge, in the mortality and complication rates between groups HHbA1c and NHbA1c $(P<.05)$, and in the values of glucose and HbAlc between groups GP and PP $(P<.05)$. The multivariate logistic linear regression analysis reveal that the neurological function changes 2 weeks after IAT were related to multiple risk factors; however, the HbA1c level (OR 0.096; $95 \%$ CI 0.545 to 0.889 ) and NIHSS score at admission (OR 0.894; $95 \%$ CI 0.814 to 0.982 ) were found to be risk factors that may affect the neurological function changes after IAT $(P<0.05)$.

In clinical settings, HbA1c levels should be considered in patients with AIS treated with IAT. In this study, patients with AIS with higher HbAlc and glucose levels had a more severe neurological impairment during the stroke and a higher incidence of a poor prognosis. As a risk factor for the neurological function recovery in patients with AIS, HbAlc levels can be used as a laboratory index for predicting poor prognosis.

The mechanism underlying the association of abnormal glucose metabolism with a poor prognosis is still unclear. The hyperglycemia-caused poor prognosis and nerve cell damage in stroke may be due to the higher extracellular glucose accumulation, extracellular acidification, or increased damage of the blood-brain barrier [Ribo 2005], which may cause cerebral edema [Bruno 2002], inhibit plasma fibrinolysis, or cause an obstruction of the capillary blood backflow [Ahmed 2010]. Furthermore, persistent hyperglycemia may increase the infarct size [Kruyt 2010]. Arnold et al [2014] reported that diabetes and hyperglycemia are independent predictors of poor prognosis after IAT and that hyperglycemia is an independent predictor of symptomatic intracranial hemorrhage. The differences between these 2 studies may be related to the different treatment time windows and the devices used for revascularization. In the acute phase of AIS, the incidence of new ischemic lesions in patients with high HbAlc levels increases [Braemswig 2017]. Not only is HbAlc an indicator of glycemic control, it is also important in patients with AIS without a history of diabetes. Therefore, HbAlc is recommended as a routine test index for patients with AIS with or without a history of diabetes for prevention of stroke recurrence [Wu 2013] or prediction of poor clinical prognosis. The 1 -year all-cause mortality rate in patients with HbA1c higher than the normal value ( $>6.5 \%$ ) increases by $7 \%$; furthermore, it doubles when the HbA1c level exceeds 7.2\% [Wu 2014].

The limitations of this study are as follows. First, this was a single-center retrospective study. Second, study grouping was based only on HbAlc levels (>6.5\%). Third, the history of diabetes in certain patients was not clear; thus, it was not set as an indicator of analysis, which may have caused certain deviations in the grouping. Fourth, the neurological function assessment was performed at the time of discharge and can be used only as a short-term prognostic judgment index; no long-term follow-up was performed. Further studies with a larger sample size should perform further grouping targeting the history of diabetes and different levels of high HbAlc so as to provide a more meaningful evidence for the long-term prognosis and prevention of stroke and to compare the possible differences among intravenous thrombolysis, IAT, and stenting.

\section{ACKNOWLEDGMENTS}

This study was funded by the General Project Foundation of Zhongshan Science and Technology Bureau, China (2015B1107).

\section{REFERENCES}

Ahmed N, Dávalos A, Eriksson N, et al. 2010. Association of admission blood glucose and outcome in patients treated with intravenous thrombolysis: Results from the Safe Implementation of Treatments in Stroke 
International Stroke Thrombolysis Register (SITS-ISTR). Arch Neurol 67:1123-1130.

Arnold M, Mattle S, Galimanis A, et al. 2014. Impact of admission glucose and diabetes on recanalization and outcome after intra-arterial thrombolysis for ischaemic stroke. Int J Stroke 9:985-991.

Braemswig TB, Nolte CH, Fiebach JB, et al. 2017. Early new ischemic lesions located outside the initially affected vascular territory appear more often in stroke patients with elevated hemoglobin (HbA1c). Front Neurol 8:606.

Bruno A, Levine SR, Frankel MR, et al. 2002. Admission glucose levels and clinical outcomes in the NINDS rt-PA Stroke Trial. Neurology 59:669-674.

Desilles JP, Meseguer E, Labreuche J, et al. 2013. Diabetes mellitus, admission glucose, and outcomes after stroke thrombolysis: A registry and systematic review. Stroke 44:1915-1923.

Gao Y, Jiang L, Wang H, et al. 2016. Association between elevated hemoglobinA1c levels and the outcomes of patients with small-artery occlusion: A hospital-based study. PLoS One 11:e0160223.

Hallevi H, Barreto AD, Liebeskind DS, et al. 2009. Identifying patients at high risk for poor outcome after intra-arterial therapy for acute ischemic stroke. Stroke 40:1780-1785.

Jauch EC, Saver JL, Adams HP Jr, et al. 2013. Guidelines for the early management of patients with acute ischemic stroke: A guideline for healthcare professionals from the American Heart Association/American Stroke Association. Stroke 44:870-947.

Jia Q, Zhao X, Wang C, et al. 2011. Diabetes and poor outcomes within 6 months after acute ischemic stroke: the China National Stroke Registry. Stroke 42:2758-2762.

Kamouchi M, Matsuki T, Hata J, et al. 2011. Prestroke glycemic control is associated with the functional outcome in acute ischemic stroke: The Fukuoka Stroke Registry. Stroke 42:2788-2794.

Kruyt ND, Biessels GJ, DeVries JH, et al. 2010. Hyperglycemia in acute ischemic stroke: Pathophysiology and clinical management. Nat Rev Neurol 6:145-155.

Lee KJ, Lee JS, Jung KH. 2018. Interactive effect of acute and chronic glycemic indexes for severity in acute ischemic stroke patients. BMC Neurol 18:105.

O'Donnell MJ, Chin SL, Rangarajan S, et al. 2016. Global and regional effects of potentially modifiable risk factors associated with acute stroke in 32 countries (INTERSTROKE): A case-control study. Lancet 388:761-765.

Pandolfi A, Giaccari A, Cilli C, et al. 2001. Acute hyperglycemia and acute hyperinsulinemia decrease plasma fibrinolytic activity and increase plasminogen activator inhibitor type 1 in the rat. Acta Diabetol 38:71-76.

Ribo M, Molina C, Montaner J, et al. 2005. Acute hyperglycemia state is associated with lower tPA-induced recanalization rates in stroke patients. Stroke 36:1705-1709.

Roquer J, Rodriguez-Campello A, Cuadrado-Godia E, et al. 2014. The role of $\mathrm{HbA1c}$ determination in detecting unknown glucose disturbances in ischemic stroke. PLoS One 9:e109960.

Wechsler LR, Roberts R, Furlan AJ, et al. 2003. Factors influencing outcome and treatment effect in PROACT II. Stroke 34:1224-1229.

Wu S, Shi Y, Wang C, et al. 2013. Glycated hemoglobin independently predicts stroke recurrence within one year after acute first-ever non-cardioembolic strokes onset in a Chinese cohort study. PLoS One 8:e80690.

Wu S, Wang C, Jia Q, et al. 2014. HbA1c is associated with increased all-cause mortality in the first year after acute ischemic stroke. Neurol Res 36:444-452. 